\title{
KAZIMIERZ BRANDYS: EN EXIL, C'EST-A-DIRE NULLE PART?
}

Christophe POTOCKI (Centre de Recherches sur les Arts et le Langage)

Kazimierz Brandys est né à Łódź en 1916, en pleine période de guerre, encore dans la Pologne de «nulle part», dans une famille bourgeoise d'ascendance juive intégrée de longue date. Il vit son enfance dans une Pologne revenue enfin d'un long siècle d'exil intérieur, et manifeste déjà une forte propension à la fabulation, annonciatrice des développements à venir. C'est ainsi qu'il joue auprès de ses camarades un rôle de meneur en prétendant appartenir à une organisation fictive, mais il se heurte finalement à la force physique d'un garçon qui l'évince. Il connaît d'autre part un instant de fêlure : une fillette le rejette parce qu'il est... Tombe alors l'invective, le mot étrange, le mot tabou, qui brusquement fait de lui un autre, un tout autre. La faille ouverte semble cependant se refermer, la fêlure se recoller.

Elle se rouvrira durant ses études de droit à l'université de Varsovie, où a été instauré comme dans la plupart des facultés un numerus clausus des étudiants juifs ainsi que leur ségrégation dans les salles de cours, dans un contexte de violences antisémites. Elle sera définitivement rouverte par un coup de canne sur la tête asséné par un étudiant d'extrême droite. D'un coup, la réalité, toute l'apparente réalité polonaise vole en éclats. Brandys analyse l'impact décisif de ce choc dans En Pologne, c'est-à-dire nulle part : « Le coup de bâton m'avait d'abord étourdi ; mais après avoir repris connaissance, j'allais commencer à voir les choses autrement. À voir mon propre pays, ma patrie réelle, en mouvement. Non plus à travers la littérature, ou à travers l'histoire. La Pologne m'a rattrapé, m'a sondé, et m'a rossé. [...] ...ce traumatisme crânien a éveillé en moi un hémisphère de conscience jusque-là assoupi »'. Dans une soudaine dés-intégration, le jeune Kazimierz est violemment renvoyé de sa polonité à ses lointaines origines "immigrées ", au peuple de l'exil ; il se découvre en partie étranger dans un pays qui est, qui lui semblait jusque-là être le sien - et dont il refuse qu'il soit le sien tel qu'il est actuellement. Dédoublé, dans une réalité elle-même

\footnotetext{
${ }^{1}$ K. Brandys, Nierzeczywistość [Irréalité], Varsovie 1977 ; paru sous le titre En Pologne, c'est-à-dire nulle part..., trad. fr. A. Lach, Paris 1978, pp. 22 et 24.
} 
dédoublée, il accède à une conscience nouvelle. Devenu autre, il aspire à l'avènement d'une autre Pologne.

Pour s'opposer à la dictature fascisante du « gouvernement des colonels $»^{2}$ et à l'ultra-nationalisme de la Pologne des dernières années d'avant-guerre - peut-être aussi pour se recoller intérieurement, pour se réunifier, pour retrouver une identité propre et une identité communautaire - le jeune Brandys adhère en 1936 à l'Union de la Jeunesse Socialiste Indépendante, tout en rédigeant des articles de critique théâtrale. La fracture reste cependant bien présente ; la guerre va la rendre plus radicale. Juif désormais, devenu étranger en son pays et menacé de mort, il se cache à Varsovie en dissimulant cette identité à laquelle on l'a réduit; quand il circule en ville, c'est en jouant le rôle de celui qu'il pensait être, celui d'un « simple » Polonais, d'un Polonais tout court - et c'est alors qu'il commence à écrire des œuvres de fiction, inspirées par la réalité vécue. Grâce à l'aide d'autres Polonais, grâce notamment à celle qui l'accompagnera toute sa vie, il échappe au ghetto et à la mort, avec un sentiment durable de culpabilité pour ne pas avoir partagé le sort d'une partie de sa famille, ni celui de toute une communauté qui est devenue plus largement la sienne, par solidarité, par identification. Avec un sentiment d'extériorité, aussi, à une communauté dont il n'a jamais partagé ni les rites ni les croyances et qui lui était, en cela, aussi « étrangère ».

Doublement étranger, il vit la guerre dans l'entre-deux, acteur de lui-même dans un étrange no man's land d'apparence irréelle qu'il continuera de parcourir et d'étudier tout au long de sa vie et de son œuvre. Mais il vit aussi dans la réalité crue de la guerre où l'homme est mis à nu, où les « véritables » identités - morales, intérieures - se révèlent, où les choix sont plus clairement définis, les valeurs plus clairement dessinées, en noir et blanc, en noir ou blanc - clarté évidente des valeurs et des choix que Brandys va ensuite regretter. La guerre offre l'expérience d'une réalité plus intense aussi, où l'on cö̈ncide dans une acuité exceptionnelle avec les moments de danger, de vie et d'action - moments rares d'entière coïncidence avec soi-même, qu'il sera si difficile ensuite de retrouver, si ce n'est peut-être dans l'écriture, ou encore dans les rêves.

Après l'insurrection et la destruction de Varsovie en 1944, après l'invasion «libératrice» des Soviétiques, la victoire sur le fascisme et la mise en place d'un pouvoir communiste, Brandys croit à la reconstruction, à la réintégration - à la construction d'une nouvelle réalité. C'est ainsi qu'il va pouvoir lui-même se reconstruire et se réintégrer, retrouver une nouvelle identité. Après plusieurs années de bannissement intérieur, de dissimulation et de dissociation, plus activement que jamais à nouveau il adhère. Il adhère au Parti, à son idéologie - et à l'Union des écrivains. Car il écrit, et publie, beaucoup, en grand activiste de l'écriture qu'il est devenu : des romans situés en temps de guerre, tout d'abord, avec une voix propre et quelques recherches formelles — Le Cheval de bois, Ville insoumise, Samson —, puis des récits socio-didactiques rédigés dans une adhérence forte aux canons du réalisme socialiste, de la critique sociale marxiste-léniniste soulignant le déterminisme historique. L'auteur sait, l'auteur décrit, l'auteur raconte, explique, critique « objectivement», en écrivant à la troisième personne bien sûr. L'auteur devient l'un des principaux écrivains officiels du système. Non seulement intégré : lu et célébré. Et disposant d'une liberté de mouvement exceptionnelle pour l'époque, qui lui permet de se rendre à plusieurs reprises à Paris - où des extraits de ses premières œuvres sont traduits et publiés.

\footnotetext{
${ }^{2}$ Gouvernement majoritairement militaire constitué par des proches du maréchal Piłsudski — principal homme d'État de la nouvelle Pologne indépendante — après sa mort en 1935.
} 
Après la mort de Staline et le dégel de 1956, le système se lézarde, pour s'ouvrir provisoirement. Comme un certain nombre de communistes croyants, Brandys ne remet pas le système en question, il en dénonce progressivement de l'intérieur les erreurs, les excès, tout en continuant d'en défendre la validité et l'existence. L'abandon de cette armature, l'arrachement à cette grande coquille si solide et le retour au dénuement est encore tout à fait impensable. II tentera plus tard d'expliquer l'aveuglement de cette foi, de cette si mauvaise foi. Brandys renonce cependant à la dictature du déterminisme et de l'objectivité, il redonne substance au sujet et rend à l'individu sa place, sa liberté de choix dans sa confrontation avec l'Histoire. II publie une série de nouvelles écrites à la première personne, dont La Défense de "Grenade", qui oppose un vieux loup dialecticien à une jeune troupe de théâtre révolutionnaire, et qui a de fortes répercussions - jusque dans la revue Les Temps Modernes, qui la publie en 1957 avec le soutien de Sartre dans un numéro spécial consacré à la Pologne ${ }^{3}$, de même qu'elle publiera peu après son roman critique, politiquement incorrect, $L a$ Mère des Rois ${ }^{4}$, dont l'impact sera également important.

Brandys découvre de plus en plus clairement la "vraie » réalité sous la réalité de façade instaurée par le régime stalinien, qu'il s'évertuait à expliquer et à défendre sans véritablement la voir. Son écriture se cherche et se décale au fur et à mesure qu'il prend distance, son rapport nouveau aux formes littéraires, aux relations entre réalité et fiction, témoigne de ses doutes, de son incertitude. Cherchant à se resituer, il perpétue l'emploi de la première personne, tout en rapprochant de plus en plus le narrateur et l'auteur, jusqu'à abandonner les genres bien définis de la nouvelle et du roman pour des textes mêlant formes et genres.

Il publie les Listy do Pani Z. [Lettres à Madame Z.], correspondance fictive écrite de l'étranger — où l'exil est longuement analysé dans ses conséquences néfastes, isolement et perte d'identité : «J'écris des lettres pour me créer un alibi, une existence. N'étant pas à Varsovie, j'ai le pénible sentiment de n'être nulle part ${ }^{5}$. Paraissent ensuite Façon d'être, monologue proche parent de La Chute de Camus, puis Joker et La Place du Marché, relevant tout à la fois de l'autobiographie, du journal, de la fiction et de l'essai.

Si la perception de la réalité peut à ce point être manipulée et déformée par le pouvoir, c'est d'abord soi-même qu'il faut tenter de connaître, par l'auto-analyse, par le regard sur soi et par le regard des autres ; c'est à travers la recherche de la vérité de soi, même projetée dans des figures fictives, qu'on a une chance d'accès moins mensonger ou moins fictif au réel, à l'Histoire — et c'est uniquement la perception qu'on a soimême du réel que l'on peut prétendre pouvoir transmettre.

C'est pour Brandys une véritable révolution copernicienne. L'individu est maintenant de plus en plus isolé dans son intériorité en partie indécidable et difficilement communicable, de plus en plus exilé au milieu d'une réalité factice ou très probablement inconnaissable, et d'une Histoire menaçante pour l'essentiel incontrôlable.

À ce renversement d'optique et d'écriture correspond bien sûr une révolution personnelle, une transformation politique progressivement assumée. Brandys quitte avec quelques autres le Parti communiste en 1966 suite à l'exclusion de Leszek Kołakowski ${ }^{6}$, et part enseigner aux États-Unis, puis à Paris à la Sorbonne, au cours de

\footnotetext{
${ }^{3}$ Les Temps Modernes 1957, $\mathrm{n}^{\circ} 132-133$.

${ }^{4}$ Les Temps Modernes 1957, $\mathrm{n}^{\circ} 141$.

${ }^{5}$ K. Brandys, Listy do Pani Z., Londres 1993, p. 31-32 (Varsovie 1957-1962, 3 vol.).

${ }^{6}$ Leszek Kołakowski, philosophe et historien des idées, fut exclu du Parti communiste pour avoir critiqué publiquement l'action du pouvoir alors qu'il était professeur à l'université de Varsovie.
} 
longs séjours qui lui permettent de prendre distance. Il va s'opposer de plus en plus clairement au pouvoir, qui va le censurer progressivement.

Il écrit en 1972 Wariacje pocztowe [Variations postales ou encore Folies postales], condensé caustique, virtuose et insensé de deux siècles d'histoire polonaise à travers la correspondance échangée par plusieurs générations de pères et de fils, dont certains sont exilés ou déportés. La dernière lettre, adressée en 1970 de Varsovie à un jeune fils qui a fui en Californie après les événements de 68, est particulièrement critique à l'égard de la Pologne contemporaine, et bien sûr très mal reçue par le pouvoir. Pour rappel, après la révolte des étudiants en 1968 et leur violente répression, le pouvoir communiste en a rejeté la responsabilité sur les Polonais d'ascendance juive, officiellement désignés comme "sionistes », et des milliers d'entre eux harcelés, chassés de leurs études, de leur travail, ont dû partir en exil. La célébrité et le statut de Brandys l'ont encore protégé, mais il n'a pu que ressentir profondément cette nouvelle persécution et ce nouvel exode.

En 1974, il publie un roman, un vrai roman, Pomyst [Le Troisième Henri, 1995], retour à la troisième personne mais au second degré, fiction sur la fiction, mise en jeu et en abyme de la fiction dans un récit mettant en scène Henri de Valois durant son bref et insupportable exil royal en Pologne, ainsi qu'un intellectuel polonais contemporain, Kruzoe, exilé aux États-Unis puis à Paris, où il enseigne à la Sorbonne une histoire totalement fictive de la littérature polonaise. Bien évidemment, l'auteur joue à se mettre ainsi lui-même en scène. Citons une nouvelle occurrence de ce qui est devenu chez lui un leitmotiv : «Il y a toujours un lieu, quelque part, où l'on peut échapper à sa propre vie. Mais se trouver en ce lieu signifie n'être nulle part $»^{7}$.

Brandys vit encore en Pologne, il peut sembler jouer avec le motif récurrent de l'exil, comme il joue avec la fiction, mais c'est dans un double mouvement de fascination et de distanciation, de conjuration, dont le retour à la forme du roman et à la troisième personne apparaît comme un effet tangible.

En 1976, il signe avec d'autres intellectuels la «Lettre des $101 »^{8}$ protestant contre le projet de modification de la Constitution polonaise. Il est censuré, puis mis à l'index, et connaît une nouvelle forme de bannissement intérieur qui s'aggrave d'année en année. Son dernier grand roman, Rondo, qui met aussi en scène les liens indissociables entre la réalité et la fiction, entre l'Histoire et les histoires, attendra six ans sa parution.

Dans sa nouvelle situation d'exclu, Brandys a rejoint une nouvelle communauté active : celle des opposants, des dissidents. Il devient l'un des premiers membres du comité de rédaction de la revue indépendante Zapis, publiée en circuit parallèle.

Il est obligé de publier maintenant ses œuvres en édition clandestine, ainsi qu'à l'étranger - à l'Institut Littéraire de Maisons-Laffitte, où il se rend personnellement — et bien sûr en traduction.

C'est ainsi qu'est publié, successivement sous ces trois formes, Nierzeczywistość (L'Irréalité), traduit en France sous le titre de En Pologne, c'est-à-dire nulle part. Dans ce monologue enregistré sur bande, tout à la fois réponse à une enquête sociologique, vraie-fausse confession et pamphlet politique, le narrateur dénonce l' "irréalité » régnant en Pologne, le mensonge politique omniprésent, utilisant en outre cyniquement

\footnotetext{
${ }^{7}$ K. Brandys, Le Troisième Henri, trad. fr. J.-Y. Erhel, Paris 1995, p. 10.

${ }^{8}$ Lettre adressée aux autorités par de grands noms de la culture polonaise pour protester contre le projet de révision de la Constitution proclamant notamment le rôle dirigeant du Parti et l'alliance indéfectible avec l'U.R.S.S.
} 
la mythologie historique et littéraire consubstantielle à l' « autre » irréalité polonaise, celle des temps d'avant.

Brandys analyse ce mélange de manipulation et de fiction créant, à force de temps, une réalité seconde qui vient doubler la très âpre réalité de la dictature parfois violente, de la pauvreté et de l'asservissement — double réalité schizophrénique d'un pays en exil, déplacé puis coupé de l'Europe depuis trente ans. Ce « nulle part » n'est plus celui de la Pologne de Jarry, c'est celui d'un no man's land entre le réel et l'irréel, entre deux réalités, entre l'Est et l'Ouest, entre vrai ou faux passé et faux présent, sans perspective d'avenir. C'est pourquoi tant de Polonais, se sentant exilés d'eux-mêmes dans leur propre pays, ont voulu émigrer, pour fuir cette fausse vie, cette nulle-vie, et tenter de se retrouver, peut-être, « quelque part ».

Brandys insiste cependant aussi sur la profonde incorporation de cette réalité et de ses strates multiples, de cette irréalité si complexe, qui le constitue, qui est son terreau de vie et d'écriture, son territoire de débats et de combats. Et depuis qu'il relate par procuration ses séjours à l'étranger, très souvent il le répète : « $\mathrm{Ne}$ pas être à Varsovie, c'est être nulle part », c'est devenir transparent, perdre son ombre et sa substance, se muer en fantôme ; un exil prolongé lui apparaît alors malgré tout comme impensable.

Dans ses Miesiace [Carnets de Varsovie], commencés fin 1978 et relatant toute la période des événements de Solidarność, il note en mars 1981, alors qu'il séjourne depuis plusieurs mois à Berlin : « J'ai quitté Varsovie depuis quatre mois. Il m'en reste encore trois. Pendant tout ce temps, je vis comme en sursis, dans un entracte de la réalité, je ne peux me défaire de cette impression. L'ordre de la journée n'a presque pas changé - travail, lectures, conversations - , cependant le sentiment d'être en-dehors $\mathrm{du}$ temps réel ne me quitte pas. Une vie factice, une pause. Et une inquiétude lancinante, jointe aux remords $»$.

Début décembre, en pleine crise politique, il part avec sa femme Maria pour un bref séjour à New York. Les Carnets s'interrompent sur ces mots : « New York, le 13 décembre 1981. L'état de guerre a été proclamé en Pologne. Toutes les communications sont coupées $\gg$.

Retourner en Pologne, ce ne serait très probablement pas la prison, mais du moins une certaine forme de claustration, une forte pression politique, la condamnation au silence. Les Brandys restent plusieurs mois à New York. Ils savent déjà qu'ils sont « tombés hors du temps », et qu'ils sont pour longtemps contraints à l'exil. Ils viennent chercher refuge à Paris, qui leur est bien plus proche, qu'ils connaissent depuis de longues années, dont ils maîtrisent la langue - et où l'auteur, plusieurs fois publié, jouit d'une certaine reconnaissance. Brandys repartira cependant à New York pour un long séjour d'enseignement à la Columbia University, et à l'âge de 66 ans, reviendra vivre définitivement à Paris.

Quelques phrases essentielles de l'auteur, programmatiques, vont déterminer tout ou presque de l'œuvre à venir. Il écrit au début des Carnets Paris-New York-Paris, en juin 1982 après sa première arrivée à Paris : «Et maintenant? Que faire ? Écrire sur tout ce qui s'est passé et qui continue de se passer, sur ce qui s'est écroulé poursuivre les Carnets ? [...] Dans mes notices de New-York, j'ai écrit : "On dit que la vie est un roman. J'écris mes Carnets pour voir si la mienne en est un.” Ce n'est pas mal. Peut-être même vrai. Mais à présent il s'agit d'autre chose. J'écris ce journal-

\footnotetext{
${ }^{9}$ K. Brandys, Carnets de Varsovie, 1978-1981, trad. fr. T. Douchy, Paris 1985, p. 216.

${ }^{10}$ Ibid., p. 286.
} 
roman parce que c'est pour moi le seul moyen de surmonter la situation d'un homme tombé hors du temps $\gg{ }^{11}$.

C'est l'exil qui conduit donc Brandys à approfondir cette nouvelle forme hybride $\mathrm{du}$ journal-roman, dans lequel il compte non seulement reprendre le fil du temps, reprendre vie, consistance, réalité, mais aussi se reconstruire, et tenter de reconstruire l'image d'une réalité polonaise dévastée par l'état de guerre - en entrelaçant la Pologne intérieure, liée à toute l'épaisseur du passé, et la Pologne contemporaine, réelle, mais perçue à distance. Double tentative de reconstruction qu'il poursuit en donnant à son journal la forme d'un récit construit, en écrivant sa vie comme un roman, qu'elle est et qu'il en fait; en s'écrivant, en se constituant en personnage d'un monde réfléchi et recréé, dans l'indissociable alliage de fiction et de réel auquel il donne un statut non plus seulement littéraire, social ou politique, mais bien ontologique. C'est une ontologie liée à « l'autre peuple du livre » qu'est la Pologne - qui a survécu à sa totale disparition en partie grâce à sa littérature. C'est l'immense impact de l'irréalité littéraire qui donnait à ce pays de nulle part l'essentiel de sa substance, de sa réalité.

Toujours installé à Paris, Brandys décide d'arrêter les Carnets en 1987. Comme on achève un roman, car un journal ne s'arrête, ne peut s'arrêter qu'avec la mort. La Pologne va bientôt regagner son indépendance, le combat de fond est terminé. Brandys peut maintenant librement retourner y vivre. II décide librement de rester. Habitué à son milieu parisien, où il a créé avec sa femme une «bulle» polonaise, iI refuse de replonger dans son ancien milieu, trop consubstantiel, trop prenant, qu'il considérait jadis comme vital et dont il a si longtemps été coupé. Ce n'est plus l'exil, c'est le retour qui lui semble maintenant impensable. Tout en visitant régulièrement son pays, il opte pour la distanciation de l'exil, ou plutôt de ce qu'il appelle maintenant la « résidence à l'étranger ", salutaire sinon féconde pour bien des écrivains, qu'ils soient ou non Polonais. Il n'est pas nulle part, il est dans l'entre-deux, comme il l'a si souvent été, dans cet entre-deux qu'il a tant expérimenté, narré, analysé - entre exils et adhésions, entre Pologne recréée et nouvelle Pologne bien réelle.

Les traductions françaises se succèdent de 1985 à 1995 : les trois volumes des Carnets ; Rondo et Le Troisième Henri ; Hôtel d'Alsace et autres adresses, recueil d'essais consacrés à Wilde, Gide et Léautaud ; L'art d'être aimée, recueil de nouvelles dialoguées retraçant diverses étapes de la Pologne depuis la guerre, dont les plus récentes, L'art de la conversation et Madame King mettent en scène à Paris, en mêlant humour et tragédie, deux personnages de femmes émigrées, de l'ancienne et de la nouvelle génération. Puis quelques années plus tard, traduit à titre posthume, un « journal-essai » intitulé De mémoire..., rédigé de façon très libre, très digressive.

Son dernier livre, non traduit en français, s'intitule Przygody Robinsona (1999) [Les Aventures de Robinson] — en écho significatif et évident au personnage de Kruzoe dans Le Troisième Henri. II y relate l'exil extrême d'un coma de plusieurs mois, dans lequel il a vécu avec un sentiment de réalité jusque-là inconnu les rêves les plus intenses, délirants ou cohérents ; il y relate aussi, ou semble y relater, les croisements de la vie et du roman, en y mêlant dans des récits apparemment véridiques la réalité et la fiction, qu'il dénonce au tout dernier moment comme telle. Nostalgie d'un vétéran du roman, nous confie-t-il... Mais en ajoutant aussitôt que pour lui cette tentative de greffe de la fiction sur les événements vraiment vécus n'a pu réussir, car il a tenté d'endosser la peau de la vraie réalité : «J'ai tenté de revenir à la fiction, mais la fiction

${ }^{11}$ K. Brandys, Carnets Paris-New York-Paris, 1982-1984, trad. fr. T. Douchy, Paris 1987, p. $13-14$. 
et la fausseté, ce sont deux choses différentes ». Co nie jest prawda. Notatki z lektur $i$ życia [Ce qui n'est pas vrai. Notes de lecture et de vie] est le titre des dernières pages de son journal publiées à titre posthume.

Kazimierz Brandys, écrivain polonais, est mort à Paris le 11 mars 2000 après y avoir séjourné 17 ans. Cela en tout cas, malheureusement, ne semble faire aucun doute. À moins... à moins que l'écrivain n'ait investi son personnage de vieil ermite errant sur les hauteurs de la «Zabersky Valley $»^{12}$, autre nom de la Vallée de la mort ? Loin, bien loin, de tous les événements en cours, en Pologne et en Navarre...

\footnotetext{
${ }^{12}$ Cf. K. Brandys, Wariacje pocztowe [Variations postales], Varsovie 1972, p. 140.
} 